\title{
Guest editorial: Special issue based on the LION 4 conference
}

\author{
Christian Blum • Roberto Battiti
}

Published online: 15 June 2011

(C) Springer Science+Business Media B.V. 2011

The series of LION conferences (LION stands for Learning and Intelligent Optimization) aims at exploring the boundaries and uncharted territories between machine learning, artificial intelligence, mathematical programming and algorithms for hard optimization problems. The main purpose of these events is to bring together experts from the above mentioned areas to discuss new ideas and methods, challenges and opportunities in various application areas, general trends and specific developments.

Following the tradition of earlier LION conferences, this special issue is dedicated to extended versions of four carefully selected papers presented at LION 4, which was held in Venice, Italy, in January of 2010.

The first paper by Matteo Gagliolo and Jürgen Schmidhuber on Algorithm Portfolio Selection as a Bandit Problem with Unbounded Losses proposes a method that learns to allocate computation time to a given set of algorithms, of unknown performance, with the aim of solving a given sequence of problem instances in a minimum time. The second article, Discovering the Suitability of Optimisation Algorithms by Learning from Evolved Instances by Kate Smith-Miles and Jano van Hemert deals with performance prediction of algorithms from a portfolio. They propose the use of an evolutionary algorithm to evolve instances that are uniquely easy or hard for each algorithm from the portfolio, thus providing a direct method for studying the relative strengths and weaknesses of each algorithm. A case study is presented based

Support has been received by grant TIN2007-66523 (FORMALISM) of the Spanish government. In addition, Christian Blum acknowledges support from the Ramón y Cajal program of the Spanish Government.

C. Blum $(\varangle)$

ALBCOM Research Group, Universitat Politècnica de Catalunya,

Barcelona, Spain

e-mail: cblum@1si.upc.edu

R. Battiti

Dipartimento di Ingegneria e Scienza dell'Informazione,

Università degli Studi di Trento, Trento, Italy

e-mail: battiti@disi.unitn.it 
on a comprehensive study of the performance of two heuristics on the travelling salesman problem. The third article on Feature extraction from optimization samples via ensemble based symbolic regression by Kalyan K. Veeramachaneni, Ekaterina Vladislavleva and Una-May O'Reilly demonstrates a means of knowledge discovery through feature extraction that exploits the search history of an optimization run. For illustration purposes, they consider a sequencing heuristic for chaining remote sensors. Finally, the last article authored by Jérémie Dubois-Lacoste, Manuel LópezIbáñez and Thomas Stützle titled Improving the Anytime Behavior of Two-Phase Local Search deals with the anytime behavior a powerful local search method for multi-objective optimization based on solving a series of scalarizations of the multiobjective problem at hand. The authors tackle the bi-objective permutation flowshop scheduling problem and the bi-objective travelling salesman problem.

In our opinion the selected papers represent a nice sample of the topics that are dealt with at LION conferences. We hope that they will serve as an incentive to attract more researchers to dedicate their efforts to those and similar topics.

Christian Blum \& Roberto Battiti Barcelona, Trento, May 2011 